\title{
Co-silencing of Birc5 (survivin) and Hspa5 (Grp78) induces apoptosis in hepatoma cells more efficiently than single gene interference
}

\author{
QIANG WANG ${ }^{1,2}$, RONG SHU $^{3}$, HUIJUN HE $^{1}$, LI WANG ${ }^{1}$, YUAN MA $^{1}$, HUIFEN ZHU $^{1}$, \\ ZHIHUA WANG ${ }^{4}$, SHUO WANG ${ }^{5}$, GUANXIN SHEN ${ }^{1}$ and PING LEI ${ }^{1}$ \\ ${ }^{1}$ Department of Immunology, Tongji Medical College, Huazhong University of Science and Technology; \\ ${ }^{2}$ Department of Immunology, Medical College of Wuhan University of Science and Technology; \\ ${ }^{3}$ Department of Anesthesiology, Hu Bei Zhong Shan Hospital; ${ }^{4}$ Department of Urology, Tongji Hospital, \\ Tongji Medical College, Huazhong University of Science and Technology, Wuhan, Hubei, P.R. China; \\ ${ }^{5}$ Lady Davis Institute, McGill University, Sir Mortimer B. Davis-Jewish General Hospital, Montreal, QC, Canada
}

Received December 23, 2011; Accepted February 28, 2012

DOI: $10.3892 /$ ijo.2012.1471

\begin{abstract}
Birc5 (previously known as survivin) is a cancerspecific protein. Due to the upregulation of its expression in various human malignancies and its key role in apoptosis, proliferation and angiogenesis, Birc5 has attracted attention as a target for anticancer therapies. In this study, when Birc5 was silenced in HepG2 cells, $29.7 \pm 3.3 \%$ cells underwent apoptosis as expected. It was found that the expression levels of glucoseregulated protein 78 (Hspa5, previously known as Grp78) was increased by almost 3-fold in Birc5-silenced HepG2 cells. Hspa5, a master regulator of the anti-apoptotic unfolded protein response signalling network, can also promote tumor proliferation, survival and metastasis. Hence, we hypothesized that the co-silencing of Birc5 and Hspa 5 may exert a stronger apoptosisinducing effect than single gene interference. To verify this, the expression levels of Birc5 and Hspa5 in human hepatocellular carcinoma tissues were determined. Immunohistochemical staining showed that the expression of Birc5 and Hspa5 was elevated in 28 out of 31 samples. Additionally, plasmid-based siRNA against Birc5 and/or Hspa 5 were constructed and transfected into the human hepatocellular liver carcinoma cell line, HepG2. Compared with the HepG2 cells, in which Birc5 or Hspa 5 were silenced alone, only $44.2 \pm 3.4 \%$ of the co-silenced cells proliferated, and $40.3 \pm 3.7 \%$ co-silenced cells underwent apoptosis $(\mathrm{p}<0.05)$. Furthermore, tumor formation from inoculated subcutaneous co-silenced cells in nude mice was inhibited
\end{abstract}

Correspondence to: Dr Ping Lei, Department of Immunology, Tongji Medical College, Huazhong University of Science and Technology, Wuhan, Hubei 430030, P.R. China

E-mail: adaleip@sina.com

Key words: Birc5 (survivin), Hspa5 (Grp78), HepG2, RNA interference, apoptosis significantly. The current study suggests that Birc5 and Hspa5 could be important survival factors for hepatoma carcinoma cells and that the simultaneous knockdown of Birc5 and Hspa5 is more effective in inducing apoptosis in HepG2 cells than the knockdown of Birc 5 or Hspa 5 alone. The co-silencing of Birc 5 and Hspa 5 could be warranted for cancer therapy.

\section{Introduction}

Birc5 is a member of the inhibitors of apoptosis (IAPs) family. Unlike other IAPs, Birc5 is a bifunctional protein that functions as a key regulator of mitosis and inhibitor of programmed cell death. Its marked expression in cancer as opposed to normal tissue (1) and the fact that its upregulated expression correlates with tumor resistance to chemotherapy $(2,3)$, increased tumor grade, recurrence risk and decreased cancer patient survival rate, have made Birc5 a promising target for anticancer interventions (4-6). Certain in vitro and in vivo studies have indicated that the downregulation of Birc5 can sensitize human tumor cells to conventional chemotherapeutic drugs, including Taxol, doxorubicin, etoposide and cisplatin $(7,8)$. We previously reported that Birc5 ribozyme and siRNA resulted in the inhibition of both the transcription and expression of Birc5 in tumor cells, triggering cell apoptosis $(9,10)$. However, Birc5 antagonists, at least those tested so far in clinical and preclinical studies, have not shown ideal outcomes in inducing cancer cell apoptosis. The details of the multiple pathways emanating from the Birc5 networks are yet to be fully elucidated.

It has been shown that the association of Birc5 with the heat shock protein (Hsp) is required for its stability and function. For example, Hsp90 binds to the mature form of Birc5 to stabilize it in cells $(11,12)$. The targeted antibody-mediated disruption of the Birc5-Hsp90 complex in cancer cells could result in proteasomal degradation of Birc5, mitochondrial dependent apoptosis and mitotic arrest (13). Various studies have investigated the possibility of targeting Birc5 using Hsp90 inhibitors. However, Cheung et al (14) showed that targeting Hsp90 with 
small molecule inhibitors induces the overexpression of Birc5 in certain cancer cell lines and subsequently enhances the ability of cell survival in drug-treated situations. This suggests that the dual inhibition of Hsp90 and Birc5 may be warranted for cancer therapy.

The glucose-regulated protein, Hspa5, belongs to the Hsp70 protein family, which is involved in a number of cellular processes that maintain the homeostasis of cells. It is strongly induced in tumors and plays critical roles in the stress of oncogenesis (15-18). We previously showed that the overexpression of Hsp70-2 protected cancer cells against apoptosis induced by hypoxia and that the upregulated expression of Hspa5 protected NIT-1 cells from death induced by streptozotocin, cytokines or cytotoxic T lymphocytes (19-21). Hspa5 may be an important survival factor for the tumor to escape immune surveillance and allow tumor cells proliferate.

We therefore hypothesized that the silencing the Birc5 and Hspa5 simultaneously could induce a stronger apoptotic effect than the silencing of Birc5 or Hspa5 alone. Therefore, the purpose of this study was to determine whether the co-silencing of Birc5 and Hspa5 is more effective in inducing apoptosis in hepatoma cells than single gene interference.

\section{Materials and methods}

Plasmid vectors. Plasmid-based siRNA against Birc5 and/or Hspa 5 was designed using the Ambion Target finder (http://www. ambion.com/techlib/misc/sirna_finder.html). A BLAST search was used for selecting two target non-cross matching sequences among the siRNA candidates generated. The 21-nucleotide target sequences of exon 1 (5'-GGACCACCGCATCTCTACATT-3') and exon 3 (5'-AGCATTCGTCCGGTTGCGCTT-3') of the human Birc5 mRNA (Genbank accession no. NM_001168.1) were selected and named as pgsiRNA-Birl and pgsiRNABir2, respectively. Both sequences were designed against all Birc5 mRNA splicing variants. The 19-nucleotide target sequence (5'-AGTGTTGGAAGATTCTGAT-3') of the human Hspa5 mRNA (Genbank accession no. NM_005347) was selected and named pgsiRNA-Hsp. The co-silencing plasmid, pgsiRNA-Bir+Hsp, was designed specifically against the exon 3 sequence of Birc5 and the above-mentioned Hspa5 sequence. The control siRNA plasmid, pgsiRNA-C, containing a sequence (5'-CGTACGCGGAATACTTCGATT-3') with no significant homology to any known human mRNA, was used throughout the study. All pGenesil plasmids (Genesil, Wuhan, China) contain genes for enhanced green fluorescent protein (EGFP) as a fluorescence marker.

Cell culture and transfection. The HepG2 human hepatocellular liver carcinoma cells were cultured in DMEM complete growth medium (Invitrogen, Carlsbad, CA, USA) supplemented with $10 \%$ fetal bovine serum (FBS; Invitrogen). Cells were maintained in a humidified $37^{\circ} \mathrm{C}$ incubator with $5 \% \mathrm{CO}_{2}$. Plasmids were transfected into HepG2 cells using Lipofectamine 2000 (Invitrogen) according to the manufacturer's instructions.

Twenty-four hours before transfection, cells (2x10 $/$ well) were plated into 6-well plates. Twelve hours before transfection, the medium was replaced by antibiotics-free medium. When cells reached $80-90 \%$ confluence, the medium was replaced with OPTI-MEM ${ }^{\circledR}$ I Reduced Serum Medium (Invitrogen).
Plasmids $(4 \mu \mathrm{g})$ complexed with $10 \mu \mathrm{l}$ of Lipofectamine ${ }^{\mathrm{TM}}$ Plus were added to the wells and incubated for $5 \mathrm{~h}$ before being replaced by fresh medium containing $10 \%$ (v/v) FBS. All the following experiments were performed at $48 \mathrm{~h}$ posttransfection.

Immunofluorescence assay. HepG2 cells were fixed in $4 \%$ paraformaldehyde for $1 \mathrm{~h}$ to detect the expression of Birc5 or Hspa5 on the membrane of HepG2 cells or were permeabilized by $0.1 \%$ saponins in PBS for 30 min after fixation to detect the expression of Birc5 or Hspa5 in the cytoplasm of HepG2 cells. Fixed cells were incubated with rabbit anti-human Birc5 (Santa Cruz Biotechnology, Santa Cruz, CA, USA) at 1:200 dilution, or goat anti-human Hspa5 (Santa Cruz Biotechnology) at 1:200 dilution. The antigen-antibody complexes were detected using FITC-conjugated goat anti-rabbit IgG (1:200 dilution; GE Healthcare UK Ltd., Buckinghamshire, UK), or donkey anti-goat IgG (1:200 dilution; GE Healthcare UK Ltd.) and the mean fluorescence intensities were determined by flow cytometry (FACSCalibur, BD Biosciences, San Jose, CA, USA) and analyzed with CellQuest software (BD Biosciences).

Immunohistochemistry. Tumor and adjacent non-tumor tissues from the primary liver cancer patients were surgically collected and immediately frozen at $-80^{\circ} \mathrm{C}$ until use. For immunohistochemical analysis, 31 liver tissues with stage I to IV hepatocellular carcinoma (HCC) (obtained from Professor Yuan Li, Department of Pathology, Tongji hospital of Huazhong University of Science and Technology, Wuhan, China) were used. Permission for the use of all of these tissues for research purposes was obtained from the Institutional Review Board of Huazhong University of Science and Technology.

Paraffin-embedded tissue sections were dewaxed by xylene and rehydrated orderly in sequential alcohols. After 3 washes, slides were immersed in $0.6 \%$ hydrogen peroxide. The sections were then subjected to incubation with rabbit anti-human Birc5 (1:200 diluation) or goat anti-human Hspa5 (1:200 dilution) for $30 \mathrm{~min}$. Then, the tissue sections were incubated with HRP conjugated goat anti-rabbit IgG (1:200 dilution; Santa Cruz Biotechnology) or HRP conjugated donkey anti-goat IgG (1:200 dilution; Santa Cruz Biotechnology, USA) for $30 \mathrm{~min}$ at room temperature and developed with diaminobenzidine (DAB) as the choromogenic agent. Counterstaining was performed with hematoxylin. The tissue sections were counterstained with hematoxylin for $30 \mathrm{sec}$, dehydrated reversely using sequential alcohols and xylene, and then mounted using Histo-mount. Images of stained tissue sections were captured using an Olympus DP70 CCD camera with an Olympus BX51 microscope. The immunohistochemical expression of Birc5 and Hspa5 was evaluated by creating a system of $10 \%$ steps (range, 0-100\%). All immunopositive tumor cells in HCC and hepatocytes in paratumor tissues were counted in at least 10 consecutive high-power fields (x400), and the scoring guideline was based on the percentage of positive cells: -, negative staining $(-10 \%)$; + , positive weak staining $(10-50 \%)$; ++ , positive strong staining (50-100\%).

Real-time PCR. Forty-eight hours after transfection, RNA was purified from HepG2 cells using TRIzol reagent (Invitrogen) according to the manufacturer's instructions. mRNA $(1 \mu \mathrm{g})$ was 
reverse-transcribed into cDNA using RevertAid (Fermentas, Vilnius, Lithuania) in a 20- $\mu$ l system.cDNA $(2 \mu \mathrm{l})$ was amplified with the SYBR-Green Master Mix (Applied Biosystems, Carlsbad, CA, USA) using the ABI 7900 Sequence Detection System (Applied Biosystems) using the following primers: Actb ( $\beta$-actin), 5'-CCTAGAAGCATTTGCGGTGG-3' (forward) and 5'-GAGCTACGAGCTGCCTG ACG-3' (reverse); Birc5, 5'-GCCCAGTGTTTCTTCTGCTTCA-3' (forward) and 5'-GC ACTTTCTCCGCAGTTTCCTC-3' (reverse); and Hspa5, 5'-GG AGGACAAGAAGGAGGACG-3' (forward) and 5'-CAGGAG TGAAGGCGACATAGG-3' (reverse). PCR was performed by using the following parameters: $50^{\circ} \mathrm{C}$ for $2 \mathrm{~min}, 95^{\circ} \mathrm{C}$ for $2 \mathrm{~min}$, and 40 cycles of $95^{\circ} \mathrm{C}$ for $15 \mathrm{sec}$ and $60^{\circ} \mathrm{C}$ for $30 \mathrm{sec}$. The values are presented as the means $\pm \mathrm{SD}$.

Western blot analysis. Whole cell lysates at $48 \mathrm{~h}$ after transfection were loaded on $10 \%$ sodium dodecyl sulfate (SDS) polyacrylamide gels. The transferred membrane was then incubated with 1:200 diluted rabbit anti-human Birc5, or goat anti-human Hspa5. The antigen-antibody complexes were detected using HRP-conjugated anti-rabbit IgG (1:500 dilution, GE Healthcare UK Ltd.), or anti-goat IgG (1:500 dilution, GE Healthcare UK Ltd.), and visualized using ECL system (GE Healthcare UK Ltd.). ACTB ( $\beta$-actin) (rabbit polyclonal antibody at 1:200 dilution, Santa Cruz Biotechnology) signals were used to normalize the Hspa5 and Birc5 signals. Relative amounts of protein were quantified using Multi Gauge Ver2.2 image analyzing software (Fuji Photo Film Co. Ltd., Tokyo, Japan).

Proliferation assay. Effects of transfection on cellular proliferation were determined using the MTT staining method. Cells in the logarithmic growth phase were plated in 96-well plates and incubated for $24 \mathrm{~h}$ in fresh medium. The cells were then transfected with siRNA plasmid for 24,48 and $72 \mathrm{~h}$, respectively. At $4 \mathrm{~h}$ prior to the end of incubation, $20 \mu \mathrm{l}$ MTT was added to each well, followed by $150 \mu \mathrm{l}$ DMSO to terminate the reaction. The optical density (OD) of each well was measured using a microculture plate reader at wave length of $492 \mathrm{~nm}$. The cell proliferation rate (PR) was calculated using the following equation: $\mathrm{PR}=\mathrm{OD}$ value in the treated samples/OD value in the control samples x $100 \%$.

Apoptosis analysis. Forty-eight hours after transfection, cells were collected, washed twice with PBS and resuspended in APC-labeled Annexin V (BD Biosciences) and PI according to the manufacturer's instructions. Cells were then incubated for $20 \mathrm{~min}$ in the dark. Cell-associated fluorescence was analyzed using WinMDI2.8 software.

Experiments in vivo. Transfected $\left(3 \times 10^{6}\right)$ HepG2 cells (dissolved in $0.2 \mathrm{ml}$ PBS) were injected subcutaneously into 5-week-old male BALB/c nude mice ( $\mathrm{n}=6$ in each group). The mice were kept in a pathogen-free environment at a constant temperature $\left(23 \pm 2^{\circ} \mathrm{C}\right)$ and humidity (50-70\%) with a 12-h light-dark cycle. Tumor sizes were measured every 3 days and calculated by the following formula: volume $\left(\mathrm{mm}^{3}\right)=1 / 2(\text { width })^{2} \mathrm{x}$ length. The growth curve of the tumor was shown.

Statistical analysis. All experiments were performed in triplicate and data were expressed as the means \pm SD. Statistical analyses
Table I. Analysis of relative Birc5 and Hspa5 expression levels between paracancerous and tumor regions by immunohistochemistry.

\begin{tabular}{|c|c|c|c|c|}
\hline \multirow[b]{2}{*}{ Array no. } & \multicolumn{2}{|c|}{ Paratumor } & \multicolumn{2}{|c|}{ Tumor } \\
\hline & Birc5 & Hspa5 & Birc5 & Hspa5 \\
\hline 1 & - & - & ++ & + \\
\hline 2 & - & + & ++ & ++ \\
\hline 3 & - & - & ++ & + \\
\hline 4 & - & - & ++ & + \\
\hline 5 & - & + & ++ & ++ \\
\hline 6 & - & - & ++ & + \\
\hline 7 & + & + & + & + \\
\hline 8 & - & - & ++ & + \\
\hline 9 & - & - & ++ & ++ \\
\hline 10 & - & - & ++ & + \\
\hline 11 & - & - & ++ & ++ \\
\hline 12 & + & + & + & + \\
\hline 13 & - & - & ++ & + \\
\hline 14 & - & - & ++ & ++ \\
\hline 15 & - & - & ++ & ++ \\
\hline 16 & - & - & ++ & + \\
\hline 17 & - & - & ++ & + \\
\hline 18 & - & - & ++ & + \\
\hline 19 & - & - & ++ & ++ \\
\hline 20 & - & - & ++ & + \\
\hline 21 & - & + & ++ & ++ \\
\hline 22 & - & - & ++ & + \\
\hline 23 & + & + & + & + \\
\hline 24 & - & - & ++ & + \\
\hline 25 & - & - & ++ & + \\
\hline 26 & - & + & ++ & ++ \\
\hline 27 & - & - & ++ & + \\
\hline 28 & - & - & ++ & + \\
\hline 29 & - & - & ++ & ++ \\
\hline 30 & - & - & ++ & + \\
\hline 31 & - & - & ++ & ++ \\
\hline
\end{tabular}

Scoring guidelines: -, negative staining; + , positive weak staining; ++ , positive strong staining.

were conducted with the Student's t-test and performed with SPSS 17.0 (SPSS Inc., Chicago, IL, USA). p-values $<0.05$ were considered to indicate statistically significant differences.

\section{Results}

siRNA-mediated silencing of Birc5 and upregulation of Hspa5. In an attempt to downregulate the expression of the antiapoptotic protein, Birc5, HepG2 cells were transfected with plasmids encoding siRNA against Birc5 exon 1 (pgsiRNA-Birl) and exon 3 (pgsiRNA-Bir2), as well as the control plasmid, pgsiRNA-C. The expression levels of Birc5 were evaluated 


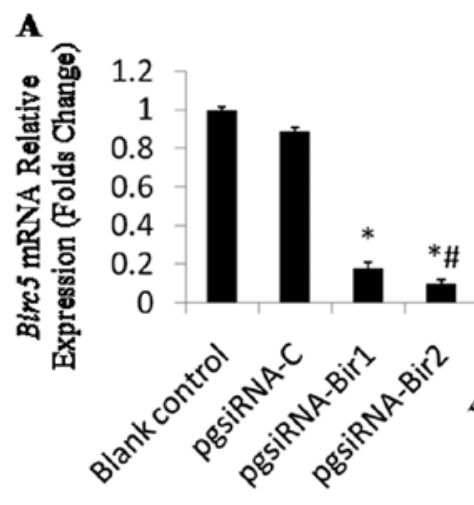

D

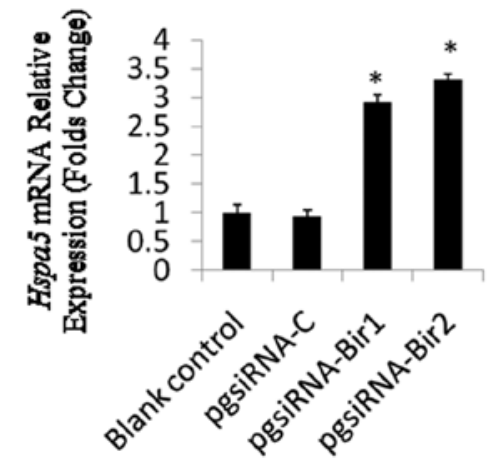

B

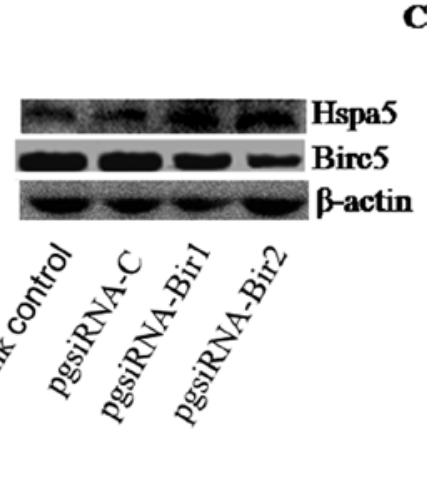

C

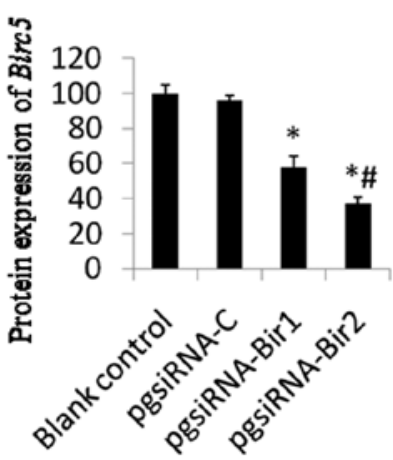

$\mathbf{E}$

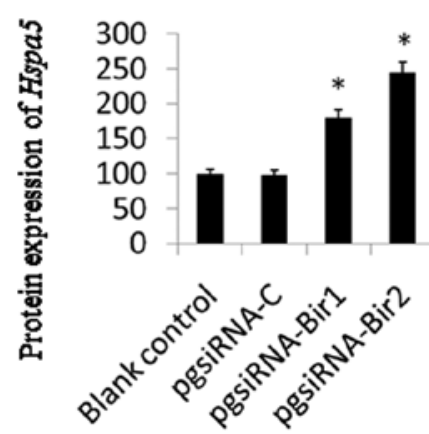

Figure 1. siRNA-mediated silencing of Birc5 and upregulation of Hspa5. (A) Birc5 mRNA levels after treatment with the control, pgsiRNA-Birl or pgsiRNABir2 detected by real-time PCR. (B) Immunoblot analysis of Birc5 and Hspa5 expression in HepG2 cells at 48 h post-transfection. (C) Densitometric analysis of Birc5 levels of the western blots. (D) Hspa5 mRNA levels after treatment with the control, pgsiRNA-Birl or pgsiRNA-Bir2 detected by real-time PCR. (E) Densitometric analysis of Hspa5 levels of the western blots. ${ }^{*} \mathrm{p}<0.05$ vs. blank control, ${ }^{\#} \mathrm{p}<0.05$ vs. pgsiRNA-Bir1.

at $48 \mathrm{~h}$ post-transfection by real-time PCR and western blot analysis. The mRNA level of Birc5 in the cells transfected with pgsiRNA-Birl or pgsiRNA-Bir2 was decreased by 18 or $10 \%$ compared to the control cells (Fig. 1A). The densitometric analysis of the western blots revealed that the protein level of Birc5 in cells transfected with pgsiRNA-Birl or pgsiRNA-Bir2 was reduced by 58 or $37 \%$ compared to the control levels, respectively, which indicated that pgsiRNA-Bir2 was more efficient than pgsiRNA-Birl (Fig. 1C).

The expression of Hspa5 was also evaluated at 48 h posttransfection by real-time PCR (Fig. 1D) and western blot analysis (Fig. 1B). The expression of Hspa5 was significantly upregulated in HepG2 cells transfected with pgsiRNA-Bir compared to the controls. For samples transfected with pgsiRNA-Birl or pgsiRNA-Bir2, the mRNA level of Hspa5 increased 2.9-fold and 3.3-fold compared to that of the non-transfected cells (Fig. 1D), respectively. Densitometric analysis revealed that the levels of Hspa5 in the cells transfected with pgsiRNA-Birl and pgsiRNA-Bir2 were increased by 1.6 - and 2.5 -fold, respectively (Fig. 1E). Transfection of pgsiRNA-C had no significant effect on HSPA5 expression.

High expression of Birc5 and Hspa5 in human liver tumors. Immunofluorescence staining showed that Birc5 and Hspa5 were highly expressed in HCC tissues, whereas were weak or undetectable in paracancerous regions (Fig. 2). Out of 31 cases, $28(90.3 \%)$ showed a higher expression of Birc5 and Hspa5 in the tumor tissue than in paracancerous areas (Table I). Only 3 (array nos. 7, 12 and 23) exhibited similar expression levels in both tumor and paracancerous regions.
Expression and cellular localization of Birc5 and Hspa5 in HepG 2 cells. Immunofluorescence assay showed that both Birc5 and Hspa5 were expressed in the cytoplasm of HepG2 cells (Fig. 3A and D). Mean fluorecence intensities (MFIs) in HepG2 cells were monitored by flow cytometry, which confirmed the observation of immunofluorescence staining (Fig. 3B and E). The MFI of Birc5 in HepG2 cells showed a significant difference ( $<<0.05$ vs. L02) (Fig. 3A and B). However, the MFI of Hspa5 in HepG2 cells showed no significant difference ( $p>0.05$ vs. L02). The Hspa5 protein was quantitatively overexpressed in HCC tumor tissues (Fig. 2B) but not in the cell lines (Fig. 3D and $\mathrm{E})$.

Knockdown of Birc5 and Hspa 5 by dual interference siRNA. The expression of Birc5 was significantly downregulated in HepG2 cells transfected with pasiRNA-Bir2 and pgsiRNA-Bir+Hsp compared to the controls. pgsiRNA-Bir2 or pgsiRNA-Bir+Hsp transfection resulted in the mRNA level of Birc5 in HepG2 cells decreasing by 14 and $15 \%$ to that of the non-transfected cells (Fig. 4A), and the protein level decreasing by 35 and $38 \%$ to that of the non-transfected cells (Fig. 4B and C). The expression of Hspa5 was also significantly downregulated in HepG2 cells transfected with pgsiRNA-Hsp and pgsiRNA-Bir+Hsp compared with the controls. After transfection by pgsiRNA-Hsp or pgsiRNA-Bir+Hsp, the HepG2 cells had a decreased mRNA expression of Hspa5 of 36 and $37 \%$ compared to that of the control cells (Fig. 4D), and a decreased protein expression of 41 and 44\% compared to that of the control cells (Fig. 4E and F), indicating that pgsiRNA-Hsp and pgsiRNA-Bir+Hsp were efficient in silencing Hspa5 gene expression (Fig. 4D). These 

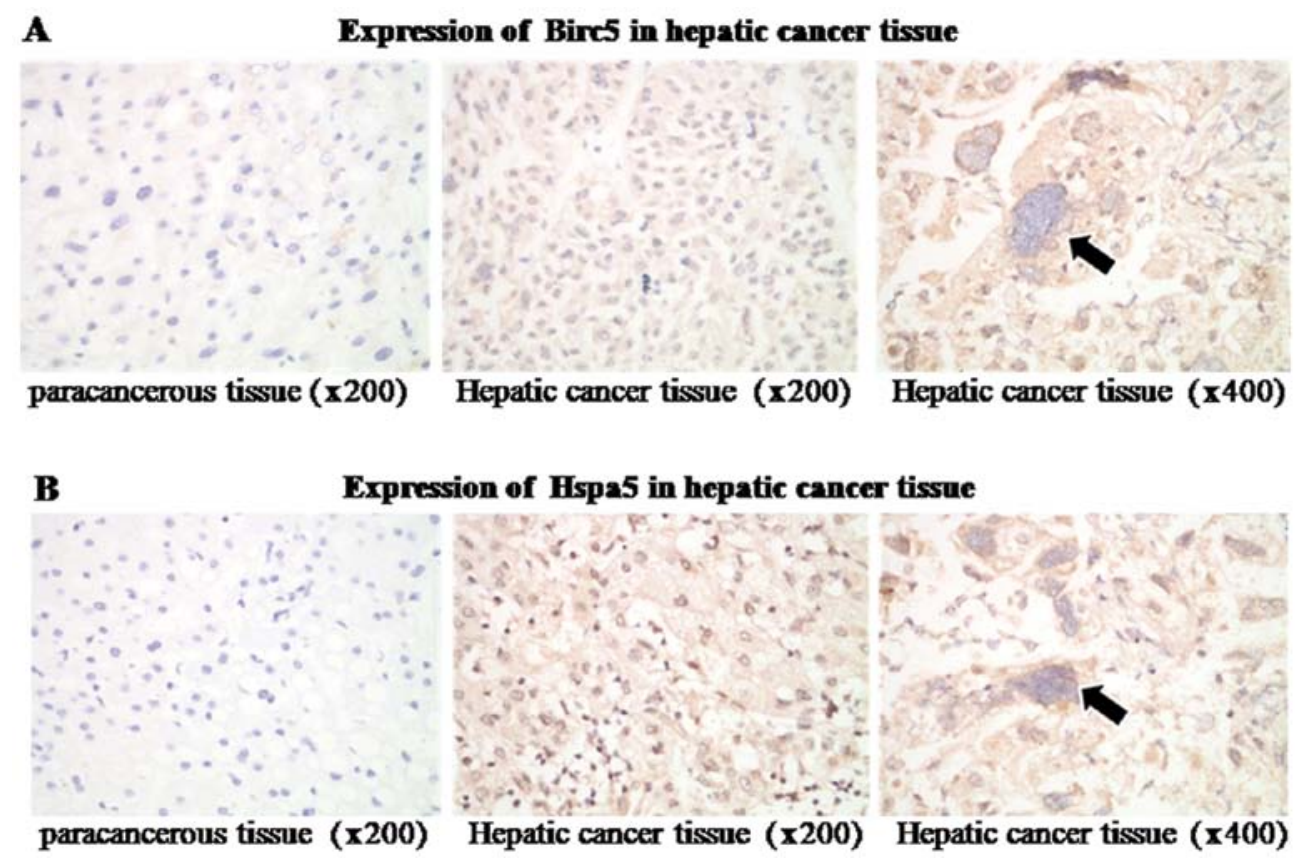

Figure 2. Immunohistochemical analysis of Birc5 and Hspa5 expression in human liver tumor samples. Paracancerous and tumor sections from paraffinembedded liver tissue array $(\mathrm{n}=31)$ were stained with anti-Birc5 or anti-Hspa5 antibody. (A) Expression of Birc5 in human liver tumors. (B) Expression of Hspa5 in human liver tumors. From left to right : paracancerous areas (x200), tumor area (x200), tumor area (x400) (black arrows indicate tumor giant cell).
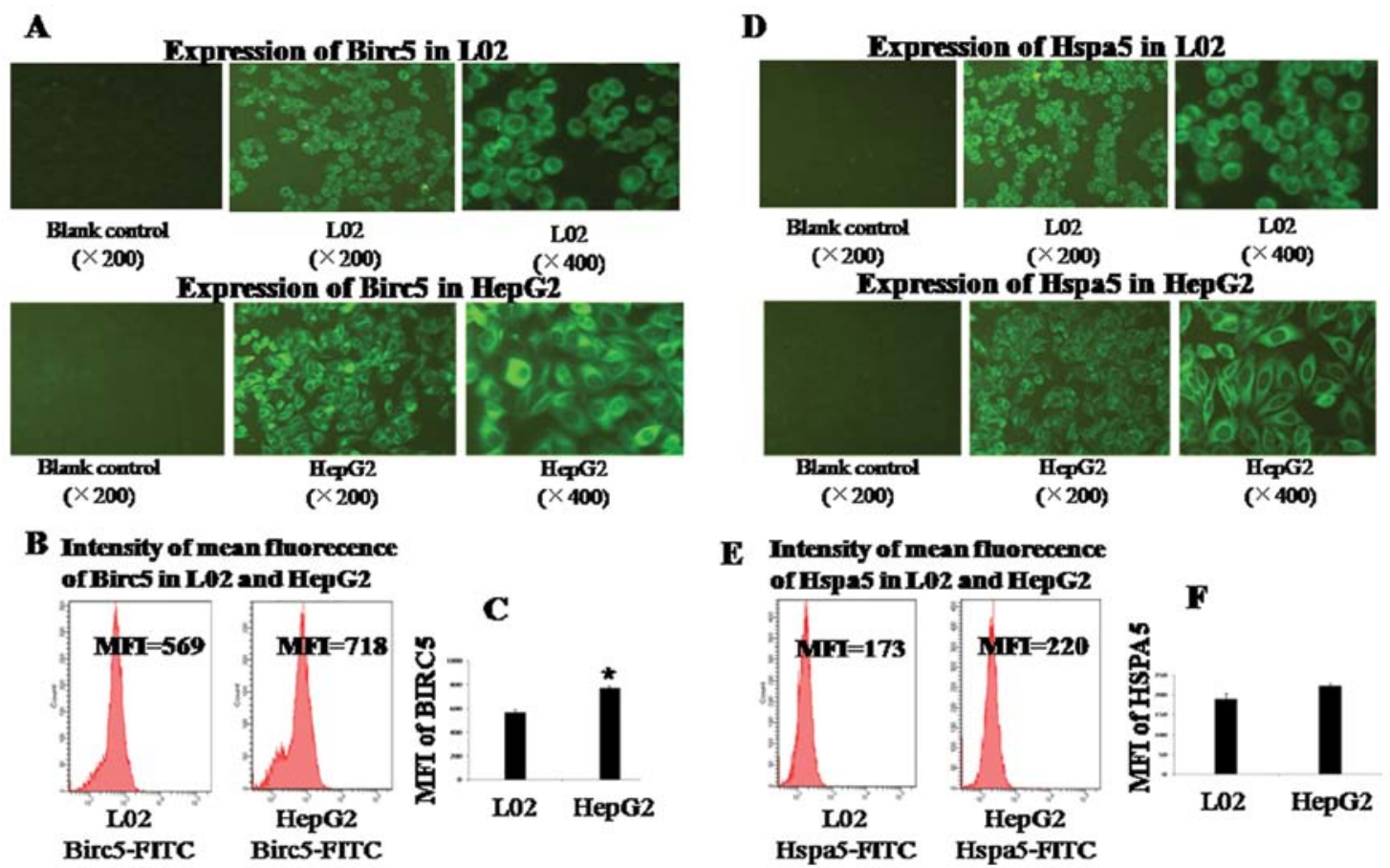

Figure 3. Expression and cellular localization of Birc5 and Hspa5 in HepG2 cells. The cells were fixed and stained with primary antibodies to (A) Birc5 and (D) Hspa5 and FITC-labeled secondary antibodies were then used (green fluorescence). (A and D) Fluorescence signals were detected by an Olympus BX51 microscope. (B and E) The intensity of mean fluorecence in HepG2 cells was monitored by flow cytometry. "p<0.05 vs. L02.

results indicate that the designed siRNAs efficiently knocked down Birc5 and Hspa5 expression in HepG2 cells.

Effect on proliferation of HepG2 cells by different siRNA plasmids. To confirm whether transfection affects the proliferation of HepG2 cells, 3-(4,5-dimethylthiazol-2-yl)-2,5-diphenyl- tetrazolium bromide (MTT) assay was used to evaluate the cell proliferation. The PRs of HepG2 cells transfected with pgsiRNA-C, pgsiRNA-Hsp, pgsiRNA-Bir or pgsiRNA-Bir+Hsp were $93.8 \pm 2.5,78.1 \pm 3.1,68.3 \pm 2.3$ and $44.2 \pm 3.4 \%$, respectively at the 48-h time-point. These data confirmed that pgsiRNA-Bir, pgsiRNA-Hsp and pgsiRNA-Bir+Hsp decreased the PR of 
$\mathbf{A}$

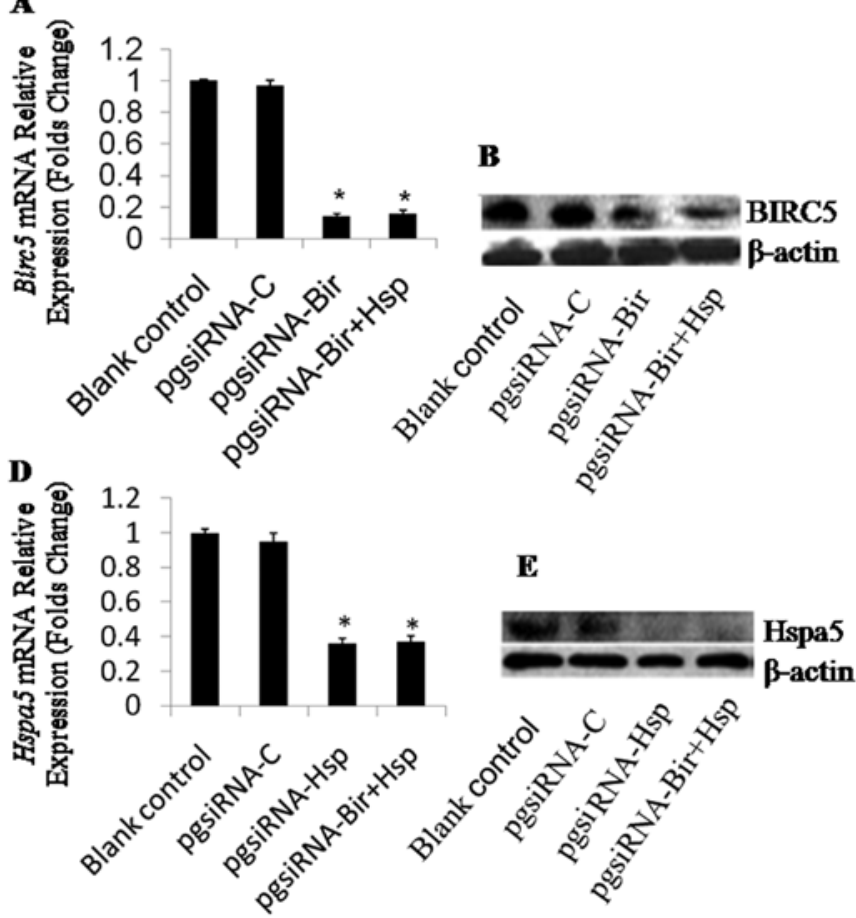

$\mathbf{C}$

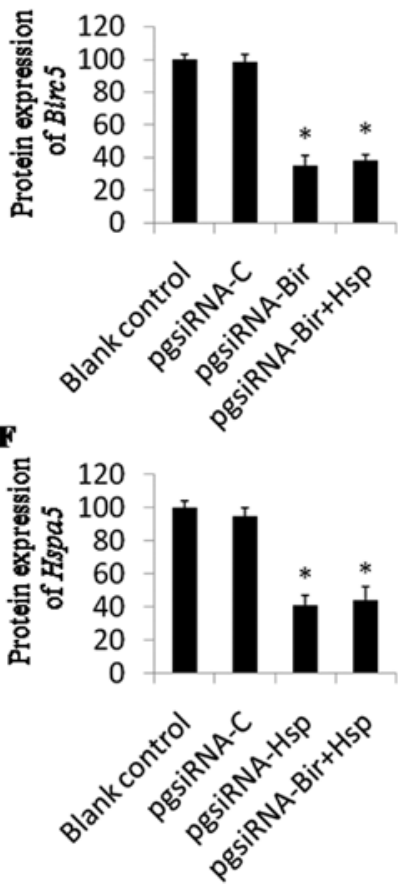

Figure 4. Silence of Birc5 and Hspa5 by dual interference siRNA plasmid. (A) Birc5 mRNA levels after treatment with control, pgsiRNA-Bir2 or pgsiRNA-Bir+Hsp detected by real-time PCR. (B) Immunoblot analysis of Birc5 expression in HepG2 cells at 48 h post-transfection of blank control, pgsiRNA-Bir or pgsiRNA-Bir+Hsp. One representative of 3 independent experiments is shown. (C) Densitometric analysis of Birc5 levels of the western blots. (D) Hspa 5 mRNA levels after treatment with control, pgsiRNA-Hsp or pgsiRNA-Bir+Hsp detected by real-time PCR. (E) Immunoblot analysis of Hspa5 expression in HepG2 cells at $48 \mathrm{~h}$ post-transfection of control, pgsiRNA-Hsp or pgsiRNA-Bir+Hsp. One representative of 3 independent experiments is shown. (F) Densitometric analysis of Hspa5 levels of the western blots. "p $<0.05$ vs. blank control.
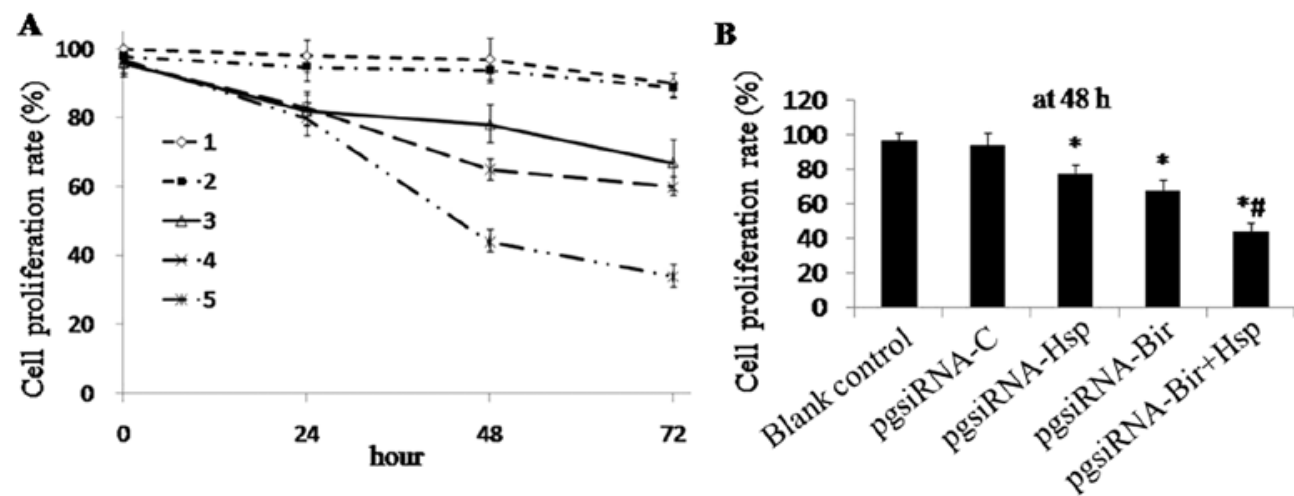

Figure 5. The effect of siRNA plasmids on proliferation of HepG2 cells. (A) HepG2 cells were treated for 24-72 h with pgsiRNA-C, pgsiRNA-Bir, pgsiRNA-Hsp or siRNA-Bir+Hsp. Proliferation assay was used to determine the growth rate relative to the control cells. 1, Blank control group; 2, pgsiRNA-C group; 3, pgsiRNA-Hsp group; 4, pgsiRNA-Bir group; 5, pgsiRNA-Bir+Hsp group; ${ }^{*} \mathrm{p}<0.05$ compared with the blank control group, ${ }^{\# p}<0.05$. compared with the pgsiRNA-Hsp and pgsiRNA-Bir groups. (B) The proliferation rates of HepG2 cells transfected with pgsiRNA-C, pgsiRNA-Hsp, pgsiRNA-Bir or pgsiRNA-Bir+Hsp at the 48 -h time-point. " $\mathrm{p}<0.05$ vs. blank control, ${ }^{\#} \mathrm{p}<0.05$ vs. pgsiRNA-Hsp and pgsiRNA-Bir.

HepG2 cells significantly compared with the controls $(\mathrm{p}<0.05)$ (Fig. 5A and B). Among these, pgsiRNA-Bir+Hsp showed the most significant anti-proliferative effect.

Co-silencing of Birc5 and Hspa5 results in increased HepG2 cell apoptosis. Flow cytometry analysis revealed that the apoptotic rates of HepG2 cells transfected with pgsiRNA-C, pgsiRNA-Hsp, pgsiRNA-Bir or pgsiRNA-Bir+Hsp were $6.3 \pm 1.5,23.1 \pm 2.1,29.8 \pm 3.3$ and $40.2 \pm 3.7 \%$, respectively, whereas only $3.0 \pm 1.5 \%$ of non-transfected cells underwent apoptosis at the 48-h time-point (Fig. 6). pgsiRNA-Hsp, pgsiRNA-Bir and pgsiRNA-Bir+Hsp significantly induced apoptosis in HepG2 cells ( $<<0.05$ vs. blank control) and pgsiRNA-Bir+Hsp was the most effective in inducing apoptosis ( $\mathrm{p}<0.05$ vs. pgsiRNA-Hsp or pgsiRNA-Bir) (Fig. 6A and B).

Inhibitory effect of co-silencing of Birc5 and Hspa5 in vivo. With the above findings of the effects of pgsiRNA-Bir+Hsp 
$\mathbf{A}$

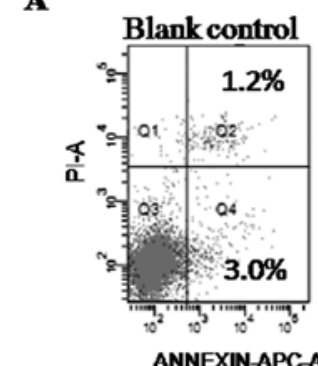

pgsiRnA-C

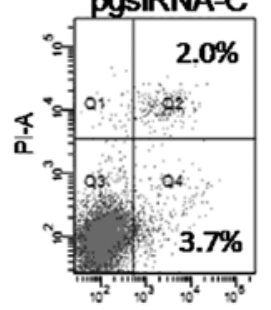

ANNEXIN-APCA
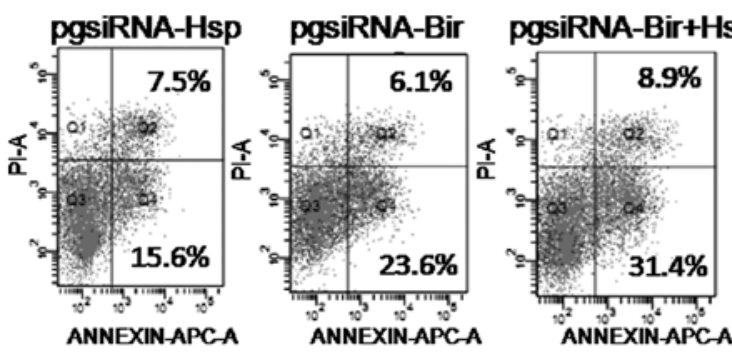

$\mathbf{B}$

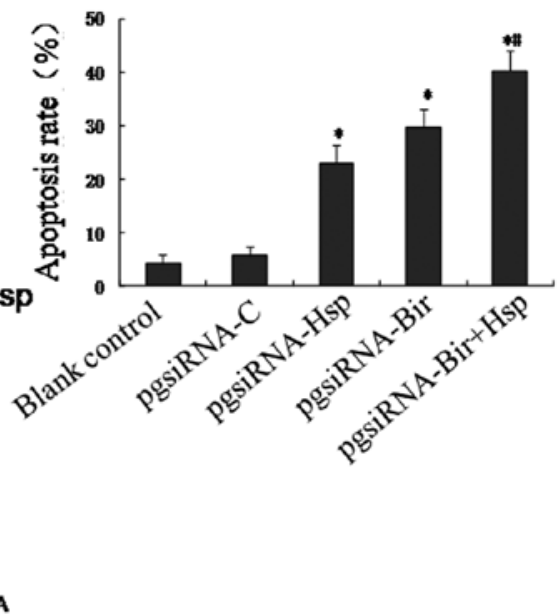

Figure 6. siRNA-mediated Birc5 and Hspa5 silencing results in HepG2 cell apoptosis. (A) Apoptotic rates of HepG2 cells treated with control siRNA, pgsiRNA-Bir, pgsiRNA-Hsp and pgsiRNA-Bir+Hsp. Forty-eight hours after transfection, the apoptosis of HepG2 cells was monitored using Annexin V-APC/ PI double-labeling followed by flow cytometry. Three parallel experiments were performed and the apoptotic rates are represented in the upper right and lower right quadrants.(B) Bars represent the average apoptotic rates of each group. ${ }^{*} \mathrm{p}<0.05$ vs. blank control group, ${ }^{\sharp} \mathrm{p}<0.05$ vs. pgsiRNA-Hsp and pgsiRNA-Bir group.

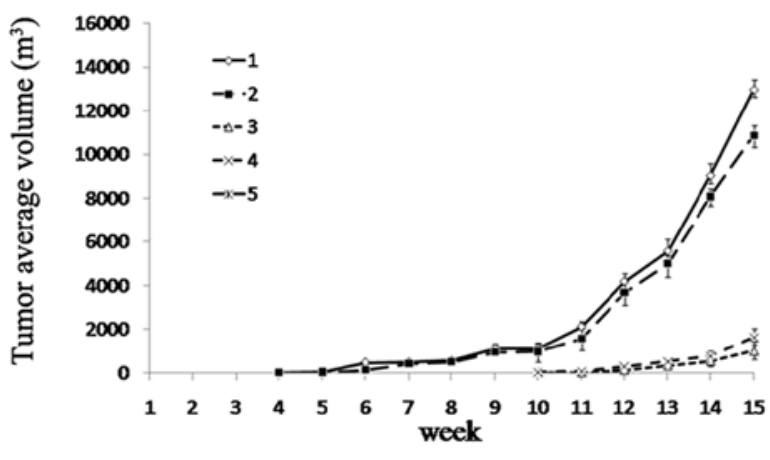

Figure 7. Tumor growth curves of transplanted HepG2 cells. 1, Blank control group; 2, pgsiRNA-C group; 3, pgsiRNA-Bir group; 4, pgsiRNA-Hsp group; 5 , pgsiRNA-Bir+Hsp group. Data are expressed as the means \pm SD.

in vitro, we next examined whether pgsiRNA-Bir+Hsp plays a critical role in tumor formation in vivo, and whether it can be used in clinical gene therapy. Equal numbers of HepG2 cells transfected with pgsiRNA-C, pgsiRNA-Bir, pgsiRNA-Hsp and pgsiRNA-Bir+Hsp, and untreated cells were injected subcutaneously into nude mice. In mice inoculated with untreated cells or pgsiRNA-C-transfected cells, tumors formed 4 weeks later. However, in those mice inoculated with cells transfected with pgsiRNA-Bir, pgsiRNA-Hsp, tumors formed 10 weeks later (Fig. 7). Furthermore, in the inoculated co-silenced cells, tumor formation in nude mice was not observed. These results indicate that the co-silencing of Birc 5 and $H$ spa 5 exerts a stronger growth suppressive effect on liver cancer in vivo.

\section{Discussion}

Birc5 is considered one of the most promising cancer targets. Certain Birc5 antagonists, such as antisense oligonucleotides
LY2181308 (22), the locked nucleic acid EZN-3042 (23) and the promoter inhibitor YM155 $(24,25)$, have been used in Phase I or II clinical trials for treating HCCs, acute myeloid leukemias, melanomas and several other advanced solid tumors. siRNA is evolving as a promising strategy for cancer therapy due to its high efficiency and specificity in blocking target mRNA expression (26).

In this study, plasmid-based siRNAs specific for Birc5 were designed and transfected into HepG2 cells. It was found that siRNA targeting Birc5 exon 3 was more effective than that targeting exon 1 in inducing cell apoptosis. Nevertheless, a small portion of cells $(29.7 \pm 3.3 \%)$ underwent apoptosis. This could be due to the low transfection efficiency. However, in our previous studies, the folate receptor-mediated specific delivery of Birc 5 siRNA caused approximately $35 \%$ of $\mathrm{HeLa}$ or MCF-7 cells to become apoptotic (10). Tirrò et al (27) also reported that the viability of WRO thyroid cancer cells transfected with siRNAs for Birc5 showed no difference with that of the scramble control. When Birc5-specific siRNA was transfected into US 8-93 human sarcoma cells, only a slight increase (2-4\%) in the percentage of cells undergoing apoptosis was observed, as well as in other 4 sarcoma cell lines. Even after an apoptotic stimulus was applied, only a small percentage of cells underwent apoptosis (28). Certain studies have shown that the use of anti-Birc 5 oligonucleotides led to the same problem $(29,30)$. The reason could be that the Birc5 knockdown resulted in the upregulation of certain molecules functionally involved in survival, anti-apoptosis in the tumor, which could counteract the effect of the Birc5 antagonists. Hendruschk et al showed that the RNAi-mediated Birc5 knockdown in U373-MG cells induced the expression of hypoxia inducible factor-1a (HIF-1a) (31). Similar to HIF-1a, Hspa5 is highly induced in the presence of low oxygen levels and plays important roles in tumor angiogenesis, apoptosis resistance, tumor cell survival (17,32-34), and furthermore, 
in the stress of oncogenesis (15-18). Therefore, we wished to determine whether the Birc5 knockdown can induce the upregulation of Hspa5.

The results from our presen study demonstrate a marked increase in the Hspa5 mRNA and protein levels upon the depletion of Birc5 in HepG2 cells and a high expression of Birc5 and Hspa5 in the HCC tissues. This suggests that the RNAi-mediated Birc5 knockdown in HepG2 cells induced the upregulation of Hspa5. However, the mechanism by which the Birc5 depletion regulates the expression of Hspa5 is still under investigation.

Taking all these results into account, we hypothesized that the co-silencing of Birc5 and Hspa5 would be more effective in apoptosis induction than single gene interference. The data confirmed that Birc5 and Hspa 5 co-silencing induced an evident increase in the percentage of cells undergoing apoptosis and an evident decrease in the percentage of cell proliferation compared with the Birc5- or Hspa5-silenced cells. Tumor formation was also not observed in the nude mice inoculated with Birc5 and Hspa 5 co-silenced cells $(\mathrm{p}<0.05)$. These results suggest that the upregulation of Hspa5 which can suppress the activities of ER stress sensors for maintaining tumor growth could be a defense mechanism in response to Birc5-specific siRNAmediated apoptosis in HepG2 cells. An inhibitor of Hspa5 may be of clinical benefit by targeting the Hspa5-protected cells after Birc5 knockdown. The dual inhibition of Birc5 and Hspa5 may be warranted for cancer therapy.

The MFI of cytoplasmic expression of Hspa5 showed no significant difference between the HepG2 and L02 cells ( $>0.05$ ), which disaccorded with the expression of Hspa5 in human HCC samples. One likely explanation is that cancer cells exhibit elevated glucose metabolism with increased glycolytic activity, and solid tumors often grow faster than their blood supply. The latter creates a tumor microenvironment characterized by glucose deprivation, acidosis and severe hypoxia (34). Solid tumor cells suffer more from these microenvironmental stresses, which leads to the activation of the unfolded protein response (UPR) in cancer cells. The UPR occurs through the transcriptional and translational regulatory mechanisms that improve the capacity of the endoplasmic reticulum (ER) to fold and traffic proteins and allows the cell to survive under stress conditions (35). The widely used sentinel marker for ER stress and UPR activation is Hspa5 (36). Therefore, solid tumor cells in these major physiological conditions are more likely to overexpress Hspa5 than tumor cell lines.

To increase the transfection efficiency, a transferrin receptor (TfR)-targeted gene delivery system is now under investigation with the aim to shed light on the potential therapeutic utilities of Birc5 and Hspa5 dual-targeting interfering siRNA.

\section{Acknowledgements}

This study was supported by: the Natural Science Foundation of China (No. 81102219, 30972734), State Project on Major Infectious Diseases Prevention (No. 2012ZX10002006-003), the New Faculty Member Program of the Chinese Department of Education (No. 20070487103), the Fundamental Research Funds for the Central Universities (No. 2010JC022, 2011TS070) and the Natural Science Foundation of Hubei Province of China (No. 2010CDB03503).

\section{References}

1. Altieri DC: Survivin, versatile modulation of cell division and apoptosis in cancer. Oncogene 22: 8581-8589, 2003.

2. Chawla-Sarkar M, Bae SI, Reu FJ, et al: Downregulation of Bcl-2, FLIP or IAPs (XIAP and survivin) by siRNAs sensitizes resistant melanoma cells to Apo2L/TRAIL-induced apoptosis. Cell Death Differ 11: 915-923, 2004.

3. Nomura T, Yamasaki M, Nomura Y and Mimata H: Expression of the inhibitors of apoptosis proteins in cisplatin-resistant prostate cancer cells. Oncol Rep 14: 993-997, 2005.

4. Li F, Yang J, Ramnath N, Javle MM and Tan D: Nuclear or cytoplasmic expression of survivin: what is the significance? Int $\mathrm{J}$ Cancer 114: 509-512, 2005.

5. Hinnis AR, Luckett JC and Walker RA: Survivin is an independent predictor of short-term survival in poor prognostic breast cancer patients. Br J Cancer 96: 639-645, 2007.

6. Altieri DC: Validating survivin as a cancer therapeutic target. Nat Rev Cancer 3: 46-54, 2003.

7. Olie RA, Simoes-Wust AP, Baumann B, et al: A novel antisense oligonucleotide targeting survivin expression induces apoptosis and sensitizes lung cancer cells to chemotherapy. Cancer Res 60: 2805-2809, 2000.

8. Pennati M, Binda M, Colella G, et al: Ribozyme-mediated inhibition of survivin expression increases spontaneous and druginduced apoptosis and decreases the tumorigenic potential of human prostate cancer cells. Oncogene 23: 386-394, 2004.

9. Liu H, Guo S, Roll R, et al: Phi29 pRNA vector for efficient escort of hammerhead ribozyme targeting survivin in multiple cancer cells. Cancer Biol Ther 6: 697-704, 2007.

10. Li L, Liu J, Diao Z, et al: Evaluation of specific delivery of chimeric phi29 pRNA/siRNA nanoparticles to multiple tumor cells. Mol Biosyst 5: 1361-1368, 2009.

11. Pennati M, Folini M and Zaffaroni N: Targeting survivin in cancer therapy: fulfilled promises and open questions. Carcinogenesis 28 : 1133-1139, 2007.

12. Gyurkocza B, Plescia J, Raskett CM, et al: Antileukemic activity of shepherdin and molecular diversity of hsp90 inhibitors. J Natl Cancer Inst 98: 1068-1077, 2006.

13. Fortugno P, Beltrami E, Plescia J, et al: Regulation of survivin function by Hsp90. Proc Natl Acad Sci USA 100: 13791-13796, 2003.

14. Cheung $\mathrm{CH}$, Chen HH, Cheng LT, et al: Targeting Hsp90 with small molecule inhibitors induces the over-expression of the antiapoptotic molecule, survivin, in human A549, HONE-1 and HT-29 cancer cells. Mol Cancer 9: 77, 2010.

15. Tanimoto R, Sakaguchi M, Abarzua F, et al: Down-regulation of $\mathrm{BiP} / \mathrm{GRP} 78$ sensitizes resistant prostate cancer cells to gene-therapeutic overexpression of REIC/Dkk3. Int J Cancer 126: 1562-1569, 2010.

16. Schardt JA, Weber D, Eyholzer M, Mueller BU and Pabst T: Activation of the unfolded protein response is associated with favorable prognosis in acute myeloid leukemia. Clin Cancer Res 15: 3834-3841, 2009.

17. Wang Q, He Z, Zhang J, et al: Overexpression of endoplasmic reticulum molecular chaperone GRP94 and GRP78 in human lung cancer tissues and its significance. Cancer Detect Prev 29: 544-551, 2005.

18. Arnaudeau S, Arboit P, Bischof P, et al: Glucose-regulated protein 78: a new partner of p53 in trophoblast. Proteomics 9: 5316-5327, 2009.

19. Huang WJ, Xia LM, Zhu F, et al: Transcriptional upregulation of HSP70-2 by HIF-1 in cancer cells in response to hypoxia. Int $\mathbf{J}$ Cancer 124: 298-305, 2009.

20. Wang M, Zhao XR, Wang P, et al: Glucose regulated proteins 78 protects insulinoma cells (NIT-1) from death induced by streptozotocin, cytokines or cytotoxic T lymphocytes. Int J Biochem Cell Biol 39: 2076-2082, 2007.

21. Wang M, Wang P, Peng JL, et al: The altered expression of glucose-regulated proteins 78 in different phase of streptozotocinaffected pancreatic beta-cells. Cell Stress Chaperones 14: 43-48, 2009.

22. Carrasco RA, Stamm NB, Marcusson EG, et al: Antisense inhibition of survivin expression as a cancer therapeutic. Mol Cancer Ther 10: 221-232, 2011.

23. Sapra P, Wang M, Bandaru R, et al: Down-modulation of survivin expression and inhibition of tumor growth in vivo by EZN-3042, a locked nucleic acid antisense oligonucleotide. Nucleosides Nucleotides Nucleic Acids 29: 97-112, 2010. 
24. Nakahara T, Kita A, Yamanaka K, et al: Broad spectrum and potent antitumor activities of YM155, a novel small-molecule surviving suppressant, in a wide variety of human cancer cell lines and xenograft models. Cancer Sci 102: 614-621, 2011.

25. Nakahara T, Takeuchi M, Kinoyama I, et al: YM155, a novel small-molecule survivin suppressant, induces regression of established human hormone-refractory prostate tumor xenografts. Cancer Res 67: 8014-8021, 2007.

26. Ashihara E, Kawata E and Maekawa T: Future prospect of RNA interference for cancer therapies. Curr Drug Targets 11: 345-360, 2010.

27. Tirrò E, Consoli ML, Massimino M, et al: Altered expression of c-IAP1, survivin, and Smac contributes to chemotherapy resistance in thyroid cancer cells. Cancer Res 66: 4263-4272, 2006.

28. Kappler M, Bache M, Bartel F, et al: Knockdown of survivin expression by small interfering RNA reduces the clonogenic survival of human sarcoma cell lines independently of p53. Cancer Gene Ther 11: 186-193, 2004.

29. Wu YF, Liang XJ, Liu YY, et al: Antisense oligonucleotide targeting survivin inhibits growth by inducing apoptosis in human osteosarcoma cells MG-63. Neoplasma 57: 501-506, 2010.
30. Xia C, Xu Z, Yuan X, et al: Induction of apoptosis in mesothelioma cells by antisurvivin oligonucleotides. Mol Cancer Ther $1:$ 687-694, 2002.

31. Hendruschk S, Wiedemuth R, Aigner A, et al: RNA interference targeting survivin exerts antitumoral effects in vitro and in established glioma xenografts in vivo. Neuro Oncol 13: 1074-1089, 2011.

32. Kern J, Untergasser G, Zenzmaier C, et al: GRP-78 secreted by tumor cells blocks the antiangiogenic activity of bortezomib. Blood 114: 3960-3967, 2009.

33. Reddy RK, Mao C, Baumeister P, et al: Endoplasmic reticulum chaperone protein GRP78 protects cells from apoptosis induced by topoisomerase inhibitors: role of ATP binding site in suppression of caspase-7 activation. J Biol Chem 278: 20915$20924,2003$.

34. Li J and Lee AS: Stress induction of GRP78/BiP and its role in cancer. Curr Mol Med 6: 45-54, 2006.

35. Saito S and Tomida A: Use of chemical genomics in assessment of the UPR. Methods Enzymol 491: 327-341, 2011.

36. Ni M, Zhou H, Wey S, et al: Regulation of PERK signaling and leukemic cell survival by a novel cytosolic isoform of the UPR regulator GRP78/BiP. PLoS One 4: e6868, 2009. 